\title{
On the reconstruction of the transfer function for atmospherically generated seiches
}

\author{
S. Monserrat \\ Instituto Mediterráneo de Estudios Avanzados (IMEDEA), CSIC-UIB, Palma de Mallorca, Spain
}

\author{
A.B. Rabinovich \\ Tsunami Center, P.P. Shirshov Institute of Oceanology, Moscow, Russia
}

\section{B. Casas}

Departament de Física, Universitat de les Hles Balears, Palma de Mallorca, Spain

\begin{abstract}
A method recently proposed to separate source and topographic effects in observed tsunami spectra is revised and applied to atmospherically generated seiches. The method is used to investigate the origin of abnormal seiche events ('rissaga waves') recorded in the region of Ciutadella, Balearic Islands. The reconstructed open-sea source spectra and the observed atmospheric pressure spectra are combined to estimate the 'transfer function' between the atmosphere and the sea surface. This function is used to predict sea level spectra for given locations near to the coast. Theoretically computed spectra for two 'rissaga' events are in good agreement with observations.
\end{abstract}

\section{Introduction}

Open ocean long waves approaching the coast occasionally generate large amplitude seiches in certain regions. These surface waves are associated with two major sources of forcing: seismic activity (tsunamis) and atmospheric disturbances (meteotsunami). Although these waves have different origins, they are modified by local topography in a similar way and may produce similar destructive consequences near the coast. Observed sea level oscillations near the coast are produced by a combined effect of some external forcing and topography. The separation of these effects and the reconstruction of the source is a key problem in tsunami study [Murty, 1977]. To solve it Rabinovich [1997] proposed a method based on comparative analysis of tsunami and background spectra at the same locations. This approach permits removal of resonant influence of local topography and insight into the tsunami source spectra.

We propose a slightly modified method to be applied to abnormal atmospherically generated seiches (meteotsunamis). In contrast to ordinary tsunami waves, the spectrum of the initial forcing (atmospheric pressure) for these seiches is often locally known. However, the transfer function between the atmosphere and sea level is not evident, especially for regions with complicated topography. Comparing meteotsunami and background

Copyright 1998 by the American Geophysical Union.

Paper number 98GL01506.

0094-8534/98/98GL-01506\$05.00 spectra, we can isolate and remove local topographic effects and estimate this function. Subsequently, the transfer function may be used for prediction purposes.

This approach is used to investigate the generation of extreme seiches ('rissaga waves') in the area of Ciutadella Inlet (Menorca, Balearic Islands). As was shown by Monserrat et al. [1991], Gomis et al. [1993], and Rabinovich and Monserrat [1996], these seiches are induced by atmospherically generated long waves incoming from the open sea. However, the exact mechanism of their formation remains unclear. Several rissaga events recorded by a number of instruments placed in various inlets and on the shelf of Menorca Island in July-August 1997 gave us an opportunity to examine the problem and verify the proposed method.

\section{Theoretical Approach}

Supposing the instrumental noise is negligible, we may present any observed spectrum, $S_{o b s}(\omega)$, during a strong event as the sum of a spectrum related to the abnormal atmospheric disturbance, $S_{r}(\omega)$, and a spectrum associated with the background oscillations, uncorrelated with this disturbance, $S_{b}(\omega)$ :

$$
S_{\omega b s}(\omega)=S_{r}(\omega)+S_{b}(\omega) \text {. }
$$

We may assume that both the abnormal and background spectra have a mutual part, $W(\omega)$, related to the local influence of topography, and different parts, related to the open-sea sources, $E_{r}(\omega)$ and $E_{b}(\omega)$, respectively:

$$
S_{r}(\omega)=W(\omega) E_{r}(\omega), \quad S_{b}(\omega)=W(\omega) E_{b}(\omega)
$$

i.e., we present both spectra as a product of the same topographic admittance function, strongly variable in space and practically constant in time, and the corresponding source spectra, locally constant in space but significantly variable in time. This assumption, which is crucial for the proposed technique, may be verified only after obtaining the final results.

Taking into account (1) and (2), we may represent the open-sea source spectrum for abnormal events as

$$
E_{r}(\omega)=E_{b}(\omega)\left(\frac{S_{a b s}(\omega)}{S_{b}(\omega)}-1\right)
$$

where the spectral ratio $R^{j}(\omega)=S_{o b s}^{j}(\omega) / S_{b}^{j}(\omega)$, is independent of the $j$ th instrument location and en- 
tirely determined by the forcing through the relation$\operatorname{ship} R^{j}(\omega)=\left(E_{r}(\omega) / E_{b}(\omega)+1\right)$.

Assuming the initial sea level variations in the open sea are forced by the atmospheric pressure, we may express $E_{r}(\omega)$ as

$$
E_{r}(\omega)=T(\omega) P_{o}(\omega),
$$

where $P_{o}(\omega)$ is the open-sea atmospheric pressure spectrum and $T(\omega)$ is the transfer function between the atmosphere and the ocean. The transfer function depends on the direction and phase speed of the atmospheric waves, and on the bathymetry of the generation region. Theoretical estimation of $T(\omega)$ is not a trivial problem, but combining (3) and (4) we get an empirical expression:

$$
T(\omega)=(R(\omega)-1) \frac{E_{b}(\omega)}{P_{o}(\omega)}
$$

which has a natural limitation requiring that background sea level oscillations and atmospheric fluctuations are much larger than the instrumental noise level [cf. Rabinovich, 1997].

Long term bottom pressure measurements in the $\mathrm{Pa}$ cific show that the background open-ocean spectrum $E_{b}(\omega)$ is virtually universal [cf. Kulikov et al., 1983] and can be described as

$$
E_{b}(\omega)=A \omega^{-2},
$$

where $A$ is a constant increasing with decreasing water depth and depending on the atmospheric activity. So, if $P_{o}(\omega)$ is known, we can use (5) to estimate the transfer function $T(\omega)$. Unfortunately, in most cases the atmospheric spectrum in the open sea $P_{o}(\omega)$ is unknown. However, if the forcing is rather local or if the atmospheric wave is coherent over a large region we may use the inshore atmospheric pressure data $P_{c}(\omega)$ in (5) instead of $P_{o}(\omega)$.

This is probably the case for the region of Ciutadella so the 1989-1990 simultaneous measurements of sea level and atmospheric pressure at Mallorca and Menorca islands [Rabinovich and Monserrat, 1996] showed that the generation area of the open-sea long waves, inducing rissaga oscillations, is the wide shallow-water shelf between these islands. Also, it was found that during strong events the atmospheric waves become coherent over a large region including this shelf and coastal areas.

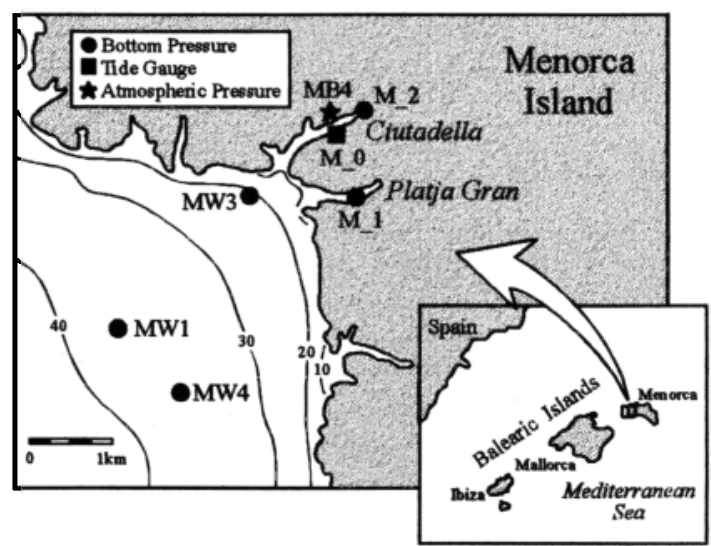

Figure 1. Location of the instruments used for the present study.

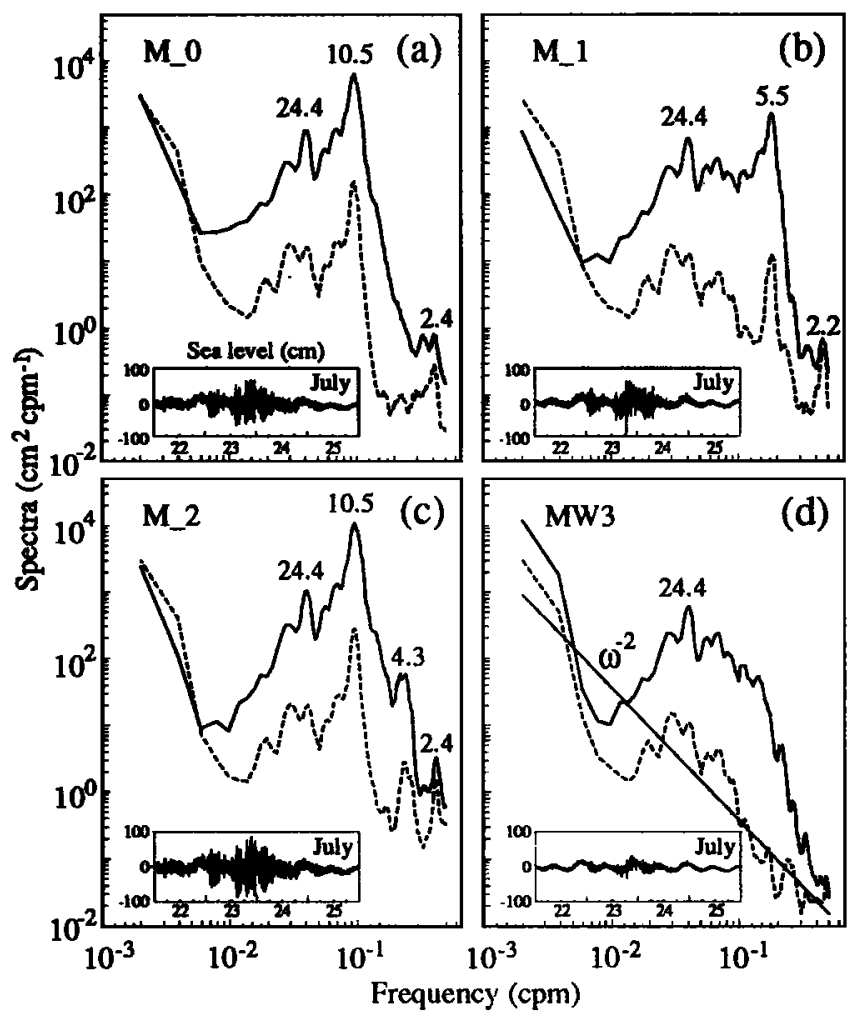

Figure 2. Sea level spectra for Rissaga 2 (solid) and for background oscillations (dashed) for the instruments M_O at the middle of Ciutadella inlet (a), M.1 at Platja Gran (b), M_2 at the end of Ciutadella inlet (c) and MW3 on the shelf. The straight line in (d) corresponds to the $\log -\log$ linear fit with a slope equal to -2 . The actual data associated with the selected four days for Rissaga 2 are shown in insets.

\section{Separation Between Source and Bathymetry}

In summer 1997, a set of precise bottom pressure recorders (BPR) and microbarographs was deployed in the region of Ciutadella, Balearic Islands (Figure 1). The BPRs had a sampling interval of $1 \mathrm{~min}$, the microbarographs were sampled every 30 sec. The instruments were placed in various inlets and on the shelf of Menorca Island, i.e. in sites with very different resonance properties. The experimental period was rich in events and several rissagas were recorded by the same instruments. The three most energetic events selected for examination are

- Rissaga 1: July 1-4, maximum height $125 \mathrm{~cm}$;

- Rissaga 2: July 23-25, maximum height $125 \mathrm{~cm}$;

- Rissaga 3: June 7-11, maximum height $110 \mathrm{~cm}$.

The same duration interval of 5760 min (4 days) was chosen for all three events. In addition, a background period of weak activity has been selected from July 27 to July 31.

Spectra of these rissaga events and background have been estimated for each instrument. A Kaiser-Bessel window of 512 points $(8.53 \mathrm{~h})$ with half-window overlapping was used to compute the averaged spectra over the 4 day period resulting in $\mathbf{4 2}$ degrees of freedom. As an example, the Rissaga 2 and background spectra for four stations are shown in Figure 2 (MW1 and MW4 


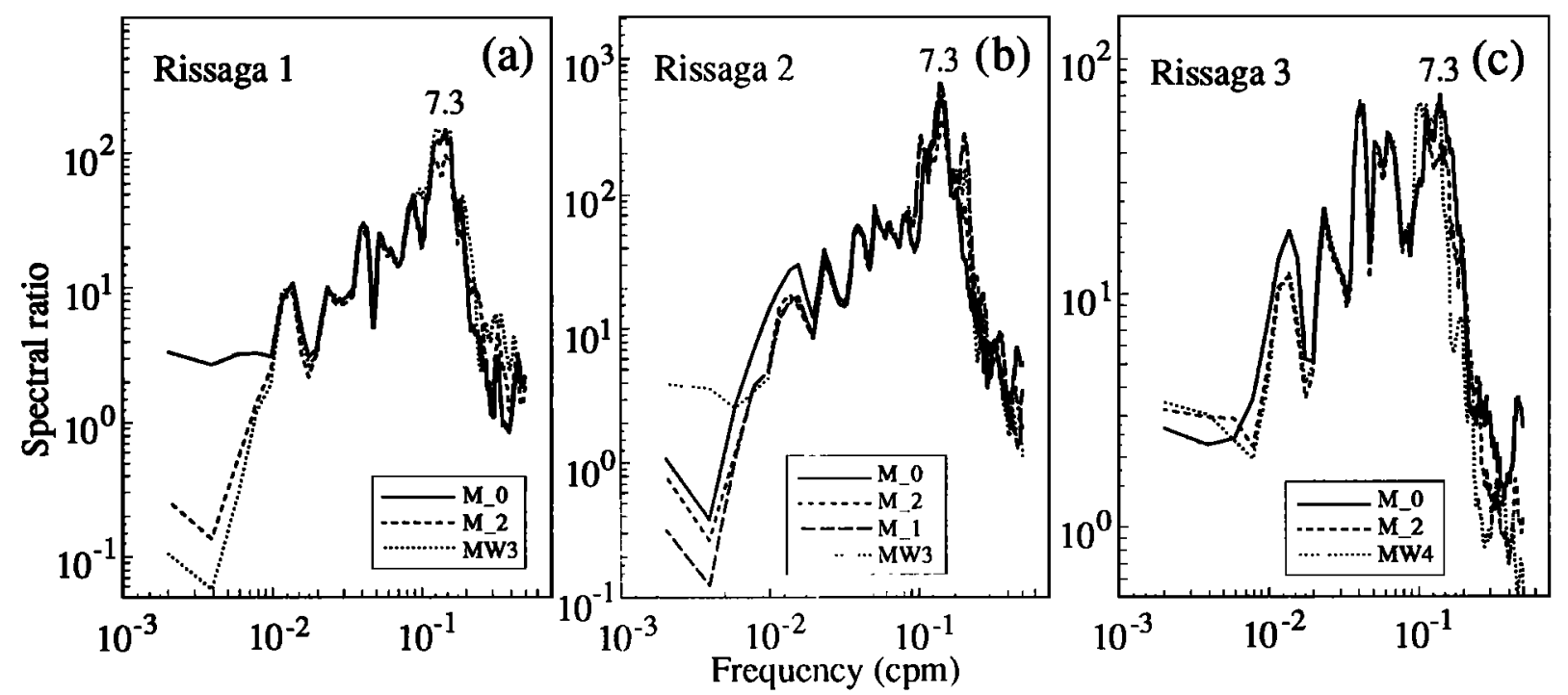

Figure 3. Spectral ratios for Rissaga 1, July 1-5 (a), Rissaga 2, July 22-25 (b) and Rissaga 3, June 7-11 (c).

spectra, which are not shown, were very similar to those for MW3). For any position the rissaga and background spectra match each other, although the rissaga spectra are much more energetic.

The spectra for different sites at high frequencies (>0.08 cpm) have significant differences, demonstrating the evident resonance influence of local topography. The only exception are stations M_0 and M_2 located in the same inlet. $A$ very energetic spectral peak with a period of $10.5 \mathrm{~min}$, related to the fundamental (Helmholtz) mode of Ciutadella Inlet, is the main feature of these spectra (Figures $2 \mathrm{a}$ and $2 \mathrm{c}$ ), in agreement with previous studies (cf. Rabinovich and Monserrat, [1996]). The first mode of the inlet (4.4 min) is seen only in the M_2 spectra, i.e. for the instrument deployed near the inlet head. The peak is absent in the M_O spectra because this instrument is located close to the nodal line of this mode. The second inlet mode of $2.4 \mathrm{~min}$ is seen for both instruments. The M_1 spectra (Figure 2b) show a fundamental mode of 5.5 min for Platja Gran Inlet, almost as energetic as the one in Ciutadella. Another mode of $2.2 \mathrm{~min}$ is also well identified for this inlet. The low frequency part of the spectra practically coincides for all instruments. Thus, the prominent peak of $24.4 \mathrm{~min}$ and some others are observed in both inlets (Figures 2a-c) and on the shelf stations MW3 (Figure 2d), MW1 and MW4 (not shown). Apparently these peaks are determined by the shelf resonance characteristics rather than by the inlet topography. Spectra for the Rissaga 1 and 3 events were very similar to those for Rissaga 2.

The spectra of the instruments located on the shelf (MW1, MW3 and MW4) were used to approximately estimate the value of $A$ in (6). The best fit gave us $A=3.710^{-3} \mathrm{~cm}^{2} \cdot \mathrm{cpm}$ for background oscillations on the shelf of Menorca, in comparison with the values $A=10^{-3}-10^{-4} \mathrm{~cm}^{2} \cdot \mathrm{cpm}$ found by Rabinovich [1997] for the open ocean and $10^{-2} \mathrm{~cm}^{2} \cdot \mathrm{cpm}$ for the Sea of Japan. The straight line with a -2 slope, adjusted to the MW3 background spectrum is shown in Figure 2d.

The different stations have quite distinctive spectra (Figure 2). However, as was previously noted, spectral ratios $R^{j}(\omega)$ are expected to be independent of the in- strument locations and entirely related to the forcing. The actual spectral ratios for the available instruments were so similar for the same events that it is difficult to distinguish them (Figure 3). This fact is a key point supporting the proposed theoretical approach and, in particular, the assumption used in (2).

The transfer functions computed for all three events using the averaged spectral ratios, $R(\omega)=\left\langle R^{j}(\omega)\right\rangle$ are shown in Figure 4. The form of the transfer functions is similar for all cases, suggesting that different rissaga events have a similar generation mechanism. This is in agreement with previous studies showing that the main characteristics of the atmospheric waves (direction, phase speed) during various observed rissaga events are very much alike [Monserrat et al., 1991; Garcies et al., 1996].

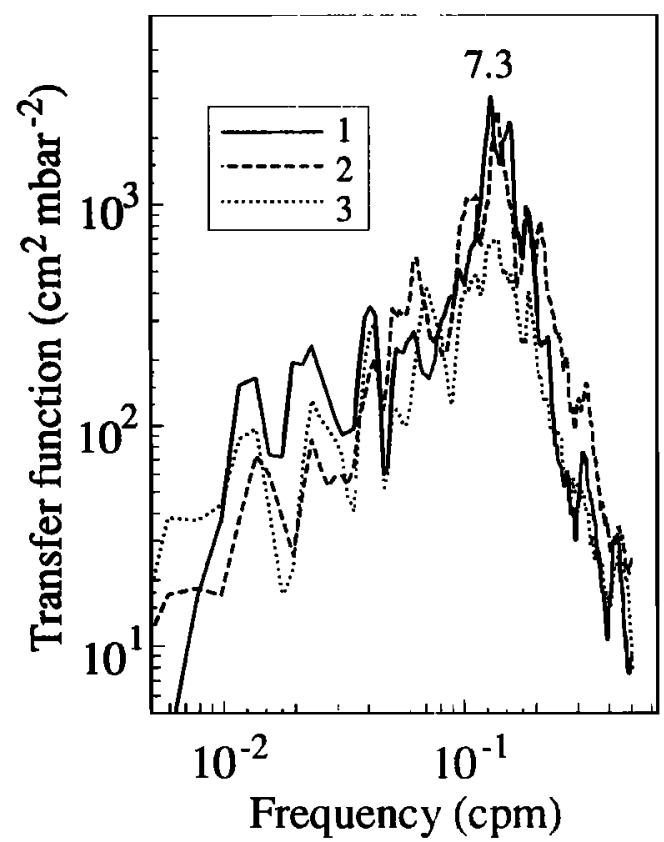

Figure 4. Averaged transfer functions between sea level and atmospheric pressure for events 1,2 , and 3 : 


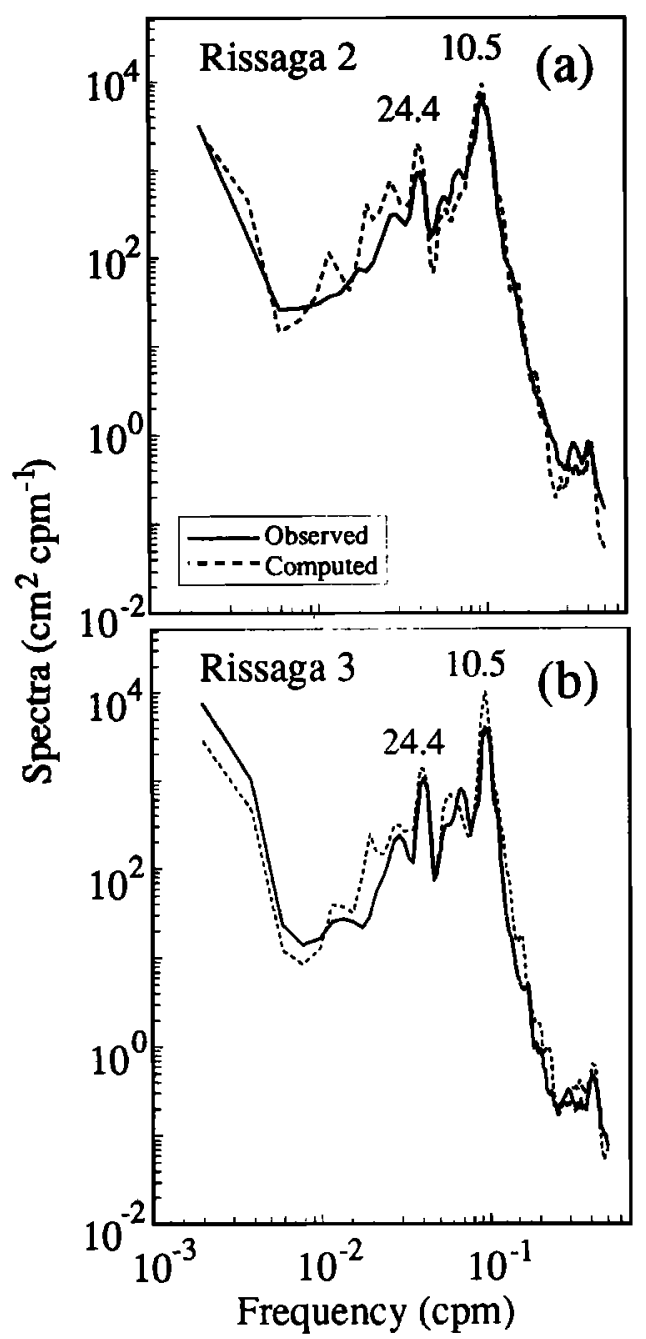

Figure 5. Observed (solid) and computed (dashed) spectra for the instrument M_O in the middle of Ciutadella inlet for Rissaga 2 (a) and Rissaga 3 (b).

Rearranging (5), we may represent the observed spectrum $S_{o b d}^{j}(\omega)$ at the $j$ th location as

$$
S_{o b s}^{j}(\omega)=S_{b}^{j}(\omega)+T(\omega) P_{c}(\omega) W^{j}(\omega),
$$

where $W^{j}(\omega)=S_{b}^{j}(\omega) / E_{b}(\omega)$. Assuming that rissaga waves are generated in a similar way for different events we may use the transfer function obtained for one event to reproduce sea level spectra for the other events by using the atmospheric pressure spectra only. As an example, we computed spectra for the M_o location for Rissagas 2 and 3 using the transfer function estimated from Rissaga 1. The agreement between computed and observed spectra is quite good (Figure 5). The major peaks, as expected, are well represented in the computed spectra, since they are mainly related to the resonant influence of the inlet topography. Furthermore, other peculiarities of the observed spectra are also reproduced by the computations. For example, the spectral peak associated with the fundamental mode, 10.5 min, is narrower for Rissaga 3 than for Rissaga 2. Also the secondary peak with a period of $15 \mathrm{~min}$ is quite evident for case 3 and almost disappears for case 2 .

Such good agreement suggests that the detailed structure of sea level spectra near the coast, although mainly related to the local topographic features, is also significantly affected by the external forcing. In particular, it clearly demonstrates that different rissaga events have very similar generation mechanisms and that the forcing is local. This local forcing justifies both the use of the same $W(\omega)$ for rissaga and background oscillations and the use of $P_{c}(\omega)$ instead of $P_{o}(\omega)$ in (5). Apparently this method will yield good results also for other regions with local character of meteotsunami generation, but will probably need certain modifications for regions with far-field sources of the induced seiches, or for regions where significant seiches are produced by some other mechanisms (wind waves, internal waves, etc.) Another important factor which may also influence the results is the non-stationary character of the signal, this question needs a separate study.

Acknowledgments. The authors would like to thank Frank Gonsales (PMEL, Seattle, WA), and Falconer Henry (IOS, Sidney, BC) for their helpful comments. The data used in this study were obtained as a collaboration with the Universidad de Cantabria and the Instituto Español de Oceanografia. This research has been partially sponsored by the CICYT of Spain under the contracts MAR95-1863 and AMB95-1251E. Financial support from INTAS-RFBR 01000196 and MAS3-CT95-0037 is gratefully acknowledged.

\section{References}

Garcies, M., D. Gomis, and S. Monserrat, Pressure-forced seiches of large amplitude in inlets of the Balearic Islands. 2. Observational study, J. Geophys. Res., 101(C3), 64536467, 1996.

Gomis, D., S. Monserrat, and J. Tintoré, Pressure-forced seiches of large amplitude in inlets of the Balearic Islands, J. Geophys. Res., 98(C8), 14,437-14,445, 1993.

Kulikov, E.A., A.B. Rabinovich, A.I. Spirin, S.L. Poole, and S.L. Soloviev, Measurement of tsunamis in the open ocean, Mar. Geodesy, 6, 311-329, 1983.

Monserrat, S., A. Ibbetson, and A.J. Thorpe, Atmospheric gravity waves and the Rissaga phenomenon, Q. J. R. Meteorol. Soc., 117, 553-570, 1991.

Murty, T.S. Seismic Sea Waves - Tsunamis, Bull. Fish. Res. Board Canada, Ottawa, 337 p., 1977.

Rabinovich, A.B., Spectral analysis of tsunami waves: Separation of source and topography effects, J. Geophys. Res., 102(C6), 12,663-12,676, 1997.

Rabinovich, A.B., and S. Monserrat, Meteorological tsunamis near the Balearic and Kuril islands: Descriptive and statistical analy sis, Nat. Hazards, 13, 55-90, 1996.

S. Monserrat, and B. Casas, Universitat de les Illes Balears, Ctra. Valldemossa, km. 7.5, E-07071 Palma de Mallorca, Spain. (e-mail: dfssmt4@ps.uib.es)

A.B. Rabinovich, Tsunami Center, Nakhimovsky Prosp., 36, 117851 Moscow, Russia. (e-mail: abrOtsucen.msk.ru)

(Received December 23, 1997; revised April 6, 1998; accepted April 20, 1998.) 